\title{
PFMDR1 AND IN VIVO RESISTANCE TO ARTESUNATE-MEFLOQUINE IN FALCIPARUM MALARIA ON THE CAMBODIAN-THAI BORDER
}

\author{
ALISA P. ALKER, PHARATH LIM, RITHY SEM, NAMAN K. SHAH, PORAVUTH YI, DENIS MEY BOUTH, \\ REIKO TSUYUOKA, JASON D. MAGUIRE, THIERRY FANDEUR, FREDERIC ARIEY, \\ CHANSUDA WONGSRICHANALAI, AND STEVEN R. MESHNICK* \\ Department of Epidemiology, UNC School of Public Health, Chapel Hill, North Carolina; Pasteur Institute of Cambodia, Phnom \\ Penh, Cambodia; National Center for Parasitology, Entomology and Malaria Control (CNM), Phnom Penh, Cambodia; World \\ Health Organization, Phnom Penh, Cambodia; US Naval Medical Research Unit No. 2 (NAMRU-2), Jakarta, Indonesia; \\ NIPH/NAMRU-2 Laboratory, National Institute of Public Health (NIPH), Phnom Penh, Cambodia
}

\begin{abstract}
Artemisinin combination therapies (ACTs) have recently been adopted as first-line therapy for Plasmodium falciparum infections in most malaria-endemic countries. In this study, we estimated the association between artesunate-mefloquine therapy failure and genetic changes in the putative transporter, $p f m d r 1$. Blood samples were acquired from 80 patients enrolled in an 2004 in vivo efficacy study in Pailin, Cambodia, and genotyped for $p$ fmdr1 copy number and haplotype. Having parasites with three or more copies of $p f m d r l$ before treatment was strongly associated with recrudescence (hazard ratio $[\mathrm{HR}]=8.30 ; 95 \% \mathrm{CI}: 2.60-26.43$ ). This relationship was maintained when controlling for initial parasite density and hematocrit (HR $=7.91 ; 95 \% \mathrm{CI}: 2.38-26.29)$. Artesunate-mefloquine treatment selected for increased $p f m d r 1$ copy number, because isolates from recurrent episodes had higher copy numbers than the paired enrollment samples (Wilcoxon rank test, $P=0.040$ ). $p f m d r 1$ copy number should be evaluated further as a surveillance tool for artesunate-mefloquine resistance in Cambodia.
\end{abstract}

\section{INTRODUCTION}

One of the challenges in the treatment and control of falciparum malaria is drug resistance. The Thai-Cambodian border, in particular, is a hotspot for the emergence of antimalarial resistance: some of the earliest reports of chloroquine and pyrimethamine resistance came from this area. ${ }^{1}$ In addition, high failure rates of mefloquine monotherapy were documented in the 1990s. ${ }^{2}$

Artemisinin combination therapy (ACT) has been promoted as an effective strategy to combat the emergence and spread of resistance. ${ }^{3}$ Currently, 38 countries have adopted ACT as first-line regimens for the treatment of malaria. ${ }^{4}$ However, a recent in vivo efficacy study on Thailand's border with Cambodia reported an artesunate-mefloquine 28-day cure rate of $78.6 \% .^{5}$ In addition, an in vivo study on the Cambodian side of the border documented a 28-day cure rate of $85.7 \%$ in $2002 .^{6}$ These studies suggest declining susceptibility to the artesunate-mefloquine combination in this subregion. However, it is not yet known whether these reported ACT failures are caused by true parasite resistance.

Because antimalarial resistance can emerge quickly, surveillance is a key element to a successful malaria control program. ${ }^{7,8}$ While the gold standard for monitoring drug efficacy is the in vivo trial, molecular markers could provide an inexpensive complementary tool. Before they can be used in a particular area, the relationship between specific molecular markers and in vivo resistance must be established.

One possible molecular marker is Plasmodium falciparum multidrug resistance gene-1 ( $p f m d r 1)$. Single nucleotide polymorphisms (SNPs) and gene copy number have previously been associated with in vitro resistance to a wide array of antimalarials, including chloroquine, lumefantrine, quinine, halofantrine, mefloquine, and artesunate. ${ }^{9-16}$ However, in vitro and in vivo resistance are not always correlated. ${ }^{13}$ The purpose of this study was to determine whether pfmdr1 ge-

* Address correspondence to Steven R. Meshnick, Department of Epidemiology, UNC Chapel Hill School of Public Health, CB \# 7435, Chapel Hill, NC 27599. E-mail: meshnick@email.unc.edu netic changes are associated with in vivo evidence of resistance to artesunate-mefloquine in Cambodia.

\section{MATERIALS AND METHODS}

Clinical study. We executed this clinical study at Pailin, Cambodia, between June and September of 2004 in conjunction with the annual monitoring of antimalarial drug efficacy in Cambodia carried out by the National Malaria Control Program. The details of this study have been previously published and were based on the WHO 2003 protocol for low transmission areas. ${ }^{6,17}$ Briefly, the eligibility criteria for this study were age $>6$ years, having uncomplicated $P$. falciparum malaria with no other Plasmodium species present, having an initial parasite density $\leq 100,000$ asexual parasites/ $\mu \mathrm{L}$, having a measured axillary temperature $\geq 37.5^{\circ} \mathrm{C}$, providing informed consent (by parent or guardian, when appropriate), and willing to return for follow-up. In total, 25 children and 56 adults were enrolled.

At enrollment, a brief clinical exam was performed, and a questionnaire was administered. Approximately $2 \mathrm{~mL}$ of venous blood was collected and frozen. Subjects were treated according to the national policy: they were given $25 \mathrm{mg} / \mathrm{kg}$ mefloquine and $12 \mathrm{mg} / \mathrm{kg}$ artesunate over 3 days (Days $0-2$ ). The mefloquine was divided into two equal doses and given in the morning and evening on the day of enrollment (Day 0). The daily $4-\mathrm{mg} / \mathrm{kg}$ dose of artesunate was divided into two doses on Day 0 and given in one dose on the next 2 days. Subjects stayed at the clinic for 3-4 days (depending on when the parasites cleared) and had follow-up visits at 7, 14, 21, 28, 35 , and 42 days after the date of enrollment. People who did not return on their own were actively sought out. At these follow-up visits, temperature was measured, and thick blood smears were used to screen for peripheral parasitemia. In instances of reoccurrence of parasitemia, an additional blood sample was acquired and stored on 3M Whatman filter paper (Whatman, Middlesex, UK).

Genotyping. DNA was extracted using the Qiamp DNA mini kit (Qiagen, Hilden, Germany). Samples taken at enrollment (Day 0), Day 1, and recurrence of parasitemia were used to distinguish between recrudescence and reinfection. 
The number of variants in three polymorphic genes (msp1, msp2, and glurp) was measured using size fractionization and ethidium bromide staining. ${ }^{18,19}$ The number of unique bands was determined visually and confirmed by measuring band size using Quantity One software on Gel Doc 2000 (Bio-Rad, Hercules, CA). If the recurrence specimen contained one or more of the variants seen in the enrollment or Day 1 specimen, the infection was classified as a recrudescence. If the recurrence specimen contained no variants present in the enrollment or Day 1 sample, it was classified as a reinfection.

Enrollment and recurrence samples were genotyped at pfmdr1 codons $86,184,1,034$, and 1,042 using real-time polymerase chain reaction (PCR). A previously published protocol was followed, except that the concentration of probes was changed to $200 \mathrm{nmol} / \mathrm{L}^{20}$

Pfmdr1 copy number was assessed for all samples using a modification of two previous assays. ${ }^{11,21}$ The primers and a FAM-TAMRA (6-carboxyfluorescein 6-carboxy-tetramethylrhodamine) probe specific to a conserved region of pfmdr1 and the primers and a VIC-TAMRA (chemical structure not released by Applied Biosystems) probe specific to $\beta$-tubulin were multiplexed so that both genes could be assayed in the same well. The primers were synthesized by MWG Biotech (High Point, NC), and the probes were synthesized by Applied Biosystems (ABI, Foster City, CA).

PCR reactions were performed on either the ABI Prism 7000 or ABI Prism 7300. Each reaction consisted of $p f m d r 1$ probe $(150 \mathrm{nmol} / \mathrm{L})$, pfmdr1 primers $(300 \mathrm{nmol} / \mathrm{L}), \beta$-tubulin probe $(100 \mathrm{nmol} / \mathrm{L}), \beta$-tubulin primers $(100 \mathrm{nmol} / \mathrm{L})$, Abgene QPCR Mastermix $(1 \times)$, DNA $(2 \mu \mathrm{L})$, and water up to $25 \mu \mathrm{L}$. If amplification of a sample was unsuccessful (no amplification, replicates being $>50 \%$ different, or a copy number $<0.6$ ), it was repeated with $4 \mu \mathrm{L}$ DNA. The reaction conditions consisted of $95^{\circ} \mathrm{C}$ for 15 minutes and then 50 cycles of $95^{\circ} \mathrm{C}$ for 15 seconds and $60^{\circ} \mathrm{C}$ for 1 minute. The cycle threshold (CT) was calculated with SDS software (ABI). DNA from strains 3D7 and Dd2 were included on each plate. The 3D7 DNA was extracted from a laboratory grown culture and Dd2 DNA was acquired from MR4 (MRA-387; ATCC, Manassas, VA).

Pfmdr1 copy number was calculated according to the following formula: copy number $=\left(\mathrm{E}_{\beta \text { tubulin }}\right)^{\mathrm{CT}\left(\beta_{\text {tubulin }} /\right.}$ $\left(\mathrm{E}_{p f m d r 1}\right)^{\text {CT(pfmdr1) }}$. The efficiency (E) of $\beta$-tubulin, which was higher than that of pfmdrl, was assumed to be 2. Pfmdrl's efficiency, relative to that of $\beta$-tubulin, was calculated for each plate by assuming the 3D7 control has one pfmdrl copy. $\mathrm{Dd} 2$, an additional control, was previously determined to have approximately four $p f m d r 1$ copies.22,23 In our assay, $\mathrm{Dd} 2$ had a mean copy number of 4.27 and a coefficient of variation of $5.77 \%$.

Statistical analysis. The clinical data were entered into Excel (Microsoft, Redman, WA) and analyzed with Stata 8.2 (StataCorp., College Station, TX). The clinical outcome of each patient was classified according to the WHO protocol. ${ }^{17}$ Because there were no early treatment failures, the main outcome for this analysis was the recurrence of $P$. falciparum parasites in the peripheral blood. Recurrences of parasitemia were further categorized as reinfections or recrudescence by the msp1, msp2, and glurp genotyping.

The copy number of samples taken before treatment and those taken at recurrence were compared using the Wilcoxon signed rank test.
The relationship between molecular changes in pfmdr1 and treatment failure was estimated using survival analysis. Because the subjects were assessed at distinct time-points, the failure time is interval-censored. Discrete Cox proportional hazards model was used to take this censoring in account. This model was estimated using regression with a complementary log-log link. ${ }^{24}$ Indicator variables representing four time periods (0-21, 22-28, 29-35, and 36-42 days) were included, whereas a constant term was not estimated. All variables were assessed for the proportional hazard assumption. The main outcome was time to recrudescence. Subjects who were lost to follow-up were censored on their last visit. Subjects who developed a $P$. vivax infection or a $P$. falciparum reinfection were censored on the day of that second diagnosis. The precision of all hazard ratio estimates were evaluated by calculating the confidence limits ratio (CLR). ${ }^{25}$

The genotype at codons $86,184,1,034$, and 1,042 in $\mathrm{pfmdr} 1$ were combined into haplotypes, as previously described. ${ }^{11}$ The comparison between haplotypes I and III was made because these haplotypes had the largest sample size and because a previous in vitro study in Cambodia found haplotype III was associated with mefloquine resistance and haplotype I with mefloquine sensitivity. ${ }^{9}$ Samples that were a mixture of haplotypes I and III were coded as haplotype III. The relationship between copy number and time to recrudescence was not linear on the log hazard scale. Therefore, copy number was coded as a binary variable of less than three copies and at least three copies to reflect the relationship between copy number and time to recrudescence and to meet the proportional hazards assumption of the model.

The following covariates were evaluated as potential effect measure modifiers and confounders: previous use of antimalarials, initial parasite density, age, sex, and hematocrit. Initial parasite density was coded as a binary variable, with the cutoff point of 80,000 parasites $/ \mu \mathrm{L}$. Effect measure modification was assessed with the Wald test for the interaction term in the model ( $P \leq 0.1$ was considered significant). All non-effect measure modifiers were evaluated in a directed acyclic diagram to select a sufficient set of covariates to control for confounding. ${ }^{26}$

The population attributable rate fraction $\left(\mathrm{AF}_{\mathrm{p}}\right)$ and $95 \%$ confidence intervals (CIs) were calculated. ${ }^{27}$ The specific formula for $\mathrm{AF}_{\mathrm{p}}$ was $\left[\left(\mathrm{HR}_{\mathrm{adj}}-1\right) \times \mathrm{p}\right) / \mathrm{HR}_{\mathrm{adj}}$, where $\mathrm{HR}_{\mathrm{adj}}=$ adjusted hazard ratio, and $\mathrm{p}=$ proportion of cases that were exposed.

The relationship between molecular change in pfmdr1 and parasite clearance time was assessed using discrete Cox proportional hazards model in the same manner as described above.

Ethics. Informed consent was obtained from all adult subjects and from the parent or guardian of all minors. The pfmdr1 genotyping and data analysis were approved by the Institutional Review Boards at the United States Naval Medical Research Unit No. 2 and at UNC Chapel Hill School of Public Health. Both the genotyping and the efficacy study was approved by the National Ethics Committee for Health Research, Ministry of Health, Cambodia.

\section{RESULTS}

Efficacy study. Of the 81 people enrolled, 10 were lost to follow-up, 7 were diagnosed with $P$. vivax, and 25 people 
experienced late treatment failure between Days 14 and 42 (Figure 1). msp1, msp2, and glurp genotyping determined that 13 of the 25 recurrences were caused by recrudescence of the original infection. In the enrollment samples, there were two $m s p 1$ variants, five $m s p 2$ variants, and five glurp variants. The prevalence of the most common $m s p 1 / m s p 2 /$ glurp haplotype was $8 \%$, which implies there is a low probability of a reinfection having an identical haplotype to the original infection.

Enrollment samples were available for $p f m d r 1$ genotyping for all but one subject. This subject was a child who recrudesced on Day 14. Clinical and demographic information for the remaining 80 subjects are presented in Table 1 .

Genotyping of enrollment samples. Of the 80 enrollment samples, 75 (93.8\%) were successfully genotyped at all four pfmdr1 codons. Of the remaining five samples, genotyping of one sample was unsuccessful at pfmdr1-86, one was unsuccessful at pfmdr1-1034, and three were unsuccessful at pfmdr1-1042. Most samples had either the I or III haplotype (Table 2). pfmdr1-86-Tyr was not observed.

pfmdr1 copy number was successfully determined for all enrollment samples. The median copy number was 1.50 (range, 0.60-6.28), and 18.8\% contained three or more copies. Elevated copy number was only observed in the presence of 86-Asn, 1034-Ser, and 1042-Asn (haplotypes I and III). There was no association between copy number and pfmdr1-184 genotype (ANOVA, $F=0.51, P=0.479$ ).

Comparison of enrollment and recurrence pfmdr1 genotypes. $p f m d r 1$ copy number was determined for all recrudescent $(N=12)$ and all reinfection $(N=12)$ samples; $75.0 \%$ $(9 / 12)$ of the reinfection samples and $66.7 \%(8 / 12)$ of the recrudescent samples had a higher copy number than the corresponding enrollment sample. These differences were not statistically significant (Wilcoxon signed rank test, for recrudescent: $\mathrm{z}=1.26, P=0.209$; for reinfection: $\mathrm{z}=1.57, P=$ 0.117). However, when comparing all enrollment and recurrent samples, the difference was statistically significant (Wilcoxon signed rank test, $\mathrm{z}=2.06, P=0.040$ ). Therefore, artesunate-mefloquine treatment seems to select for increased $p f m d r 1$ copy number.

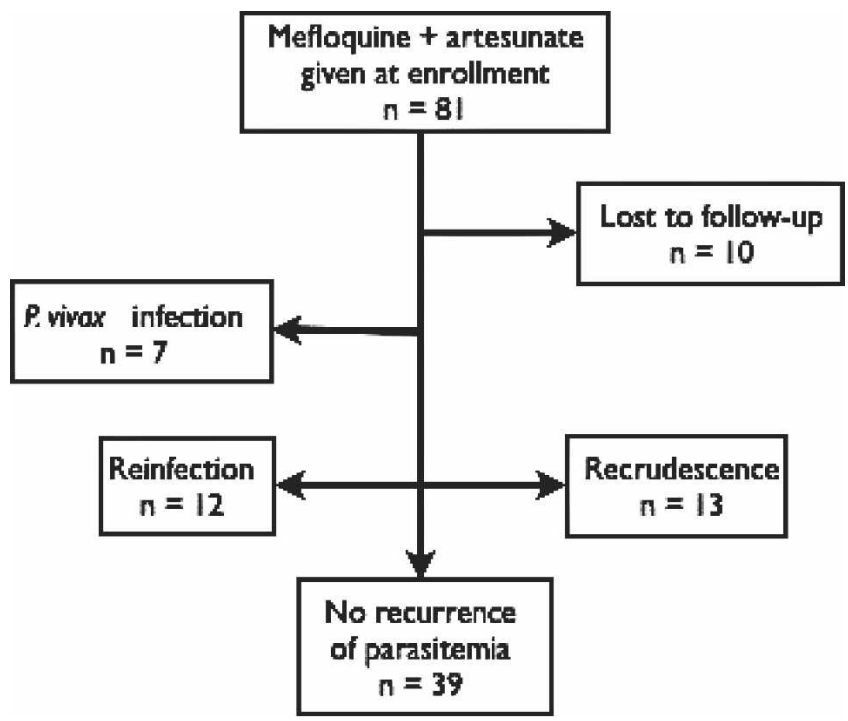

FIGURE 1. Flowchart describing the results of the in vivo efficacy trial in 2004 in Pailin, Cambodia.
TABLE 1

Clinical characteristics of the subjects in the Pailin in vivo efficacy study that were genotyped for $p f m d r 1$

\begin{tabular}{lc}
\hline \multicolumn{1}{c}{ Characteristic } & Number \\
\hline Men $(\%)$ & $57(71.3 \%)$ \\
Median age (range) & $20(6-65)$ \\
Geometric mean parasite density & \\
[parasites/ $\mu$ L (SD)] & $1.89 \times 10^{4}\left(1.16 \times 10^{5}\right)$ \\
Mean hematocrit (SD) & $38.7(5.3)$ \\
Previous use of antimalarials* $(\%)$ & $28(37.8 \%)$ \\
\hline Sample size is 80 , unless otherwise specified. & \\
$*$ *n the past month by self-report ( $=74)$. &
\end{tabular}

Four recrudescent and 10 reinfection samples were genotyped for $p f m d r 1$ polymorphisms. When comparing the haplotypes at enrollment and recurrence for the reinfection samples, five were identical, one sample changed from haplotype IV to III, two samples changed from being mixed (haplotypes I and III) to haplotype III, and two samples changed from having haplotype III to being mixed (haplotypes I and III). The four recrudescent samples had identical haplotypes to the enrollment sample (three of these paired samples had haplotype III and one had haplotype I). Thus, there seems to be no selection of a particular pfmdrl haplotype because of artesunate-mefloquine treatment.

pfmdrl and time to recrudescence. Subjects with increased pfmdrl copy number recrudesced sooner and more often than subjects with low copy number (Figure 2; Table 3). The crude hazard ratio for increased copy number (at least three copies compared with less than three copies) on time to recrudescence was 8.30 (95\% CI: 2.60-26.43). Adjusting for confounders resulted in a hazard ratio of 7.91 (95\% CI: 2.38-26.29). These results were not dependent on the statistical model, because the hazard ratio from a continuous Cox proportional model was similar in size and precision. Thus, pfmdrl copy number is strongly related to time to recrudescence.

For $p f m d r 1$ haplotype, 11 of 12 recrudescences occurred in subjects infected with parasites with haplotypes I and III. One subject with parasites with an incomplete haplotype also recrudesced. None of the four subjects with parasites with haplotype IV recrudesced; however, the small sample size prevents further evaluation of this haplotype. The crude hazard ratio of time to recrudescence comparing haplotype III to I was 2.22 (95\% CI: 0.28-17.34). Adjusting for confounders resulted in a slightly lower hazard ratio $(1.88$; $95 \%$ CI: 0.24 15.00). There seems to be little difference between haplotype III and I on time to recrudescence; however, the estimate is very imprecise $(\mathrm{CLR}=61.36)$.

To investigate the impact of increased pfmdr1 copies on recrudescence in the entire cohort, the population attribut-

TABLE 2

$p f m d r 1$ haplotypes of samples taken at enrollment with successful genotyping at all codons

\begin{tabular}{|c|c|c|c|c|c|}
\hline 86 & 184 & 1034 & 1042 & Haplotype & Number \\
\hline Asn & Tyr & Ser & Asn & I & 13 \\
\hline Asn & Phe & Ser & Asn & III & $57 \dagger$ \\
\hline Asn & Phe & Ser & Asp & IV & $4 \ddagger$ \\
\hline Asn & Tyr & Cys & Asn & V & 1 \\
\hline
\end{tabular}


A.

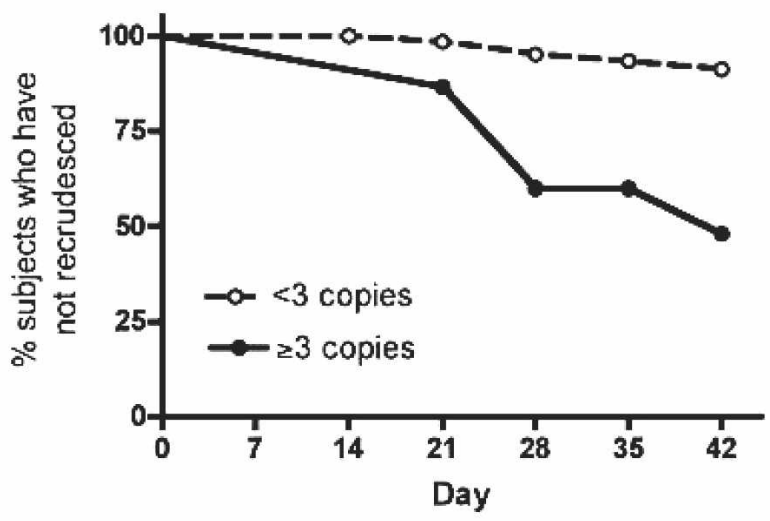

B.

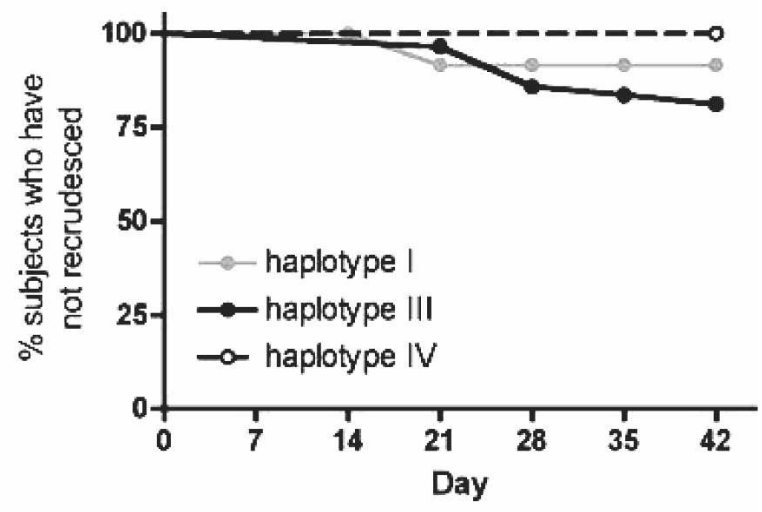

Sample size $<3$ copies:

23 copies:

$\begin{array}{rrrrrrr}65 & 65 & 65 & 64 & 62 & 50 & 44 \\ 15 & 15 & 15 & 15 & 13 & 8 & 5\end{array}$

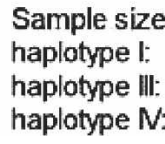

$\begin{array}{rrrrrrr}13 & 13 & 13 & 12 & 11 & 10 & 9 \\ 57 & 57 & 57 & 57 & 54 & 40 & 33 \\ 4 & 4 & 4 & 4 & 4 & 4 & 4\end{array}$

FIGURE 2. The percentage of subjects who have not recrudesced after artesunate-mefloquine treatment by $\mathbf{A}, p f m d r 1$ copy number and $\mathbf{B}$, pfmdr1 haplotype. In B, only samples with haplotypes I, III, and IV were included. The haplotypes are defined in Table 2.

able fraction was calculated. Using the prevalence of greater than three pfmdr1 copies in the cases $(58.3 \%)$ and the adjusted hazard ratio (7.91), the population attributable fraction of recrudescence for increased pfmdrl copy number was $50.9 \%$ (95\% CI: $4.1-74.9 \%)$. pfmdr1 copy number seems to contribute to the cause of one half the cases of recrudescence observed in this study.

When the outcome of all recurrences of parasites (recrudescence + reinfection) was used, the estimate of effect for copy number was lower (unadjusted $\mathrm{HR}=4.51,95 \% \mathrm{CI}$ : 1.99-10.23; adjusted HR $=4.02,95 \%$ CI: 1.73-9.34). In contrast, the hazard ratio for time to recurrence for haplotype III was higher but very imprecise (unadjusted $\mathrm{HR}=4.78,95 \%$ CI: 0.64-35.64; adjusted HR $=4.40,95 \%$ CI: 0.59-33.02). Therefore, the estimated effect of pfmdrl haplotype but not copy number changes depending on whether the treatment failures were PCR corrected. This suggests that if some misclassification of recrudescence and reinfection had occurred, the interpretation of the effects would not be substantially different.

The $p f m d r 1$ copy number is a potential screening tool for drug resistance. Having parasites with greater than three pfmdrl copies before treatment identified cases of recrudes- cence with a sensitivity and specificity of $58.3 \%$ and $88.2 \%$, respectively, and a positive predictive value of $66.7 \%$. Including information on either $p f m d r 1$ haplotype or initial parasite density increased the specificity but decreased the sensitivity of predicting recrudescence (at least three copies + haplotype III: sensitivity $=50.0 \%$, specificity $=91.2 \%$; at least three copies + parasitemia $\geq 80,000$ : sensitivity $=50.0 \%$, specificity $=91.2 \%)$.

pfmdr1 and parasite clearance time. Of the 80 subjects, 4 $(5.0 \%)$ cleared the parasitemia by Day 1, $41(51.3 \%)$ cleared by Day 2, 27 (33.8\%) cleared by Day 3, and $8(10.0 \%)$ cleared by Day 4 . The parasite clearance times observed in this study are longer than reported previously for artesunate-mefloquine. $^{28}$

Increased copy number was not associated with delayed parasite clearance when controlling for parasite density and hematocrit (Table 4). There was a slight association between pfmdrl haplotype and delayed parasite clearance time, although the estimate was imprecise.

\section{DISCUSSION}

In this study, pfmdr1 copy number at the time of treatment initiation was strongly associated with recrudescence after

TABLE 3

Hazard ratios (HRs) and $95 \%$ confidence intervals (CIs) for time to recrudescence by pfmdrl copy number and haplotype

\begin{tabular}{|c|c|c|c|c|c|c|c|c|c|}
\hline & \multirow[b]{2}{*}{ Level } & \multirow{2}{*}{$\begin{array}{l}\text { No. } \\
\text { subjects }\end{array}$} & \multirow{2}{*}{$\begin{array}{l}\text { No. } \\
\text { recrudesced }\end{array}$} & \multicolumn{3}{|c|}{ Crude hazard ratio } & \multicolumn{3}{|c|}{ Adjusted $\dagger$ hazard ratio } \\
\hline & & & & HR & $\mathrm{CI}$ & CLR* & HR & $\mathrm{CI}$ & CLR \\
\hline \multirow[t]{2}{*}{ Copy number } & $<3$ & 65 & 5 & $1 . \ddagger$ & & & 1. & & \\
\hline & $\geq 3$ & 15 & 7 & 8.30 & $2.60-26.43$ & 10.17 & 7.91 & $2.38-26.29$ & 11.04 \\
\hline \multirow[t]{2}{*}{ Haplotype } & I & 13 & 1 & 1. & & & 1. & & \\
\hline & III & 57 & 10 & 2.22 & $0.28-17.34$ & 61.93 & 1.88 & $0.24-15.00$ & 62.50 \\
\hline \multirow[t]{2}{*}{ Hematocrit } & $<40$ & 39 & 7 & 1. & & & & & \\
\hline & $\geq 40$ & 41 & 5 & 0.68 & $0.22-2.15$ & 9.78 & & & \\
\hline \multirow[t]{2}{*}{ Parasite density } & $<80,000$ & 58 & 6 & 1. & & & & & \\
\hline & $\geq 80,000$ & 22 & 6 & 3.09 & $1.00-9.61$ & 9.61 & & & \\
\hline
\end{tabular}

* Confidence limit ratio $=$ upper limit/lower limit.

$\dagger$ Adjusted for initial parasite density and hematocrit.

\$ Referent level. 
TABLE 4

Hazard ratios (HRs) and 95\% confidence intervals (CIs) for the relationship between parasite clearance time and pfmdr1 copy number and haplotype

\begin{tabular}{|c|c|c|c|c|c|c|c|c|}
\hline & \multirow[b]{2}{*}{ Level } & \multirow{2}{*}{$\begin{array}{l}\text { Median } \\
\text { PCT } \\
\text { (hours) }\end{array}$} & \multicolumn{3}{|c|}{ Crude hazard ratio } & \multicolumn{3}{|c|}{ Adjusted $\dagger$ hazard ratio } \\
\hline & & & $\mathrm{HR}$ & $\mathrm{CI}$ & CLR* & HR & $\mathrm{CI}$ & CLR \\
\hline \multirow[t]{2}{*}{ Copy number } & $<3$ & 48 & 1. & & & 1. & & \\
\hline & $\geq 3$ & 72 & 0.65 & $0.34-1.24$ & 3.65 & 0.98 & $0.48-1.98$ & 4.13 \\
\hline \multirow[t]{2}{*}{ Haplotype } & I & 48 & 1. & & & 1 & & \\
\hline & III & 48 & 0.81 & $0.40-1.63$ & 4.08 & 0.83 & $0.41-1.69$ & 4.12 \\
\hline \multirow[t]{2}{*}{ Hematocrit } & $<40$ & 48 & 1. & & & & & \\
\hline & $\geq 40$ & 48 & 1.23 & $0.76-2.00$ & 2.63 & & & \\
\hline \multirow{2}{*}{ Parasite density } & $<80,000$ & 48 & 1. & & & & & \\
\hline & $\geq 80,000$ & 72 & 0.26 & $0.14-0.49$ & 3.50 & & & \\
\hline
\end{tabular}

artesunate-mefloquine administration. After adjusting for confounding, subjects infected with parasites having greater than three copies of pfmdrl had 7.91 times the risk of recrudescence as subjects harboring parasites with less than three copies. pfmdr1 copy number was an important determinant of recrudescence, as shown by the population attributable fraction of $50.9 \%$. In addition, artesunate-mefloquine treatment was found to select for increased pfmdr1 copy number, because parasites from recurrent infections had significantly higher copy numbers than parasites from those same patients on enrollment. These data show that artesunate-mefloquine failures are associated with a specific drug-resistance biomarker and that treatment may increase the prevalence of this biomarker in the general population.

Our observed association between $p f m d r 1$ and failure is consistent with previous in vivo studies that looked at mefloquine monotherapy and artesunate-mefloquine combination therapy. ${ }^{13,21}$ In both studies, associations between pfmdr1 copy number and drug failure were found, but no association was seen between $p f m d r 1$ haplotype and drug failure. In contrast, numerous in vitro studies have found associations between both copy number and haplotype and in vitro $\mathrm{IC}_{50} \cdot{ }^{13,21,29-31}$ There are four possible reasons for this. First, the effect of these mutations might be too small to be seen in vivo. Second, determinations of SNPs in complex polyclonal infections could result in misclassification. Third, in Pailin, the pfmdrl genotype had minimal variation, with some sensitive genotypes being either rare or absent, so the effect might have been missed. Last, the factors responsible for resistance might differ by geographic region. Therefore, the inconsistency of results could be caused by the fact that several of the in vitro studies were executed outside of Southeast Asia.

It has been suggested that increased $p f m d r 1$ copy number might cause resistance to both artesunate and mefloquine. ${ }^{11-13,16}$ The observed drug failures are probably caused by mefloquine resistance, because a study in Pailin in 20012002 found the clinical isolates had high mefloquine $\mathrm{IC}_{50} \mathrm{~s}$ and low artesunate $\mathrm{IC}_{50} \mathrm{~s} .{ }^{32}$ Also, parasite clearance is predominantly caused by artesunate, and no effect of pfmdrl copy number on parasite clearance time was observed in this study. Therefore, the effect pfmdr1 copy number on recrudescence is most likely caused by the effect of $p f m d r 1$ on mefloquine sensitivity and not artesunate sensitivity. ${ }^{21}$

We observed an increase in $p f m d r 1$ copy number in parasites isolated after artesunate-mefloquine treatment. Because mefloquine has a much longer half-life than artesunate (14-41 days versus 1 hour), ${ }^{33,34}$ resistant parasites were probably selected by exposure to subtherapeutic concentrations of the single drug mefloquine. ${ }^{35}$ Selection for mefloquine resistance in Pailin may have also been facilitated by the pre-existence of resistance to this drug. ${ }^{36,37}$ This observation suggests that ACT selects for resistance to the non-artemisinin partner drug.

Strengths of this study include the fact that genotyping was done directly on peripheral blood samples, which negates the potential bias caused by culturing. ${ }^{20}$ Another strength is its 42-day follow-up with PCR correction. ${ }^{38}$ The main limitation of this study is the small sample size, which affected the power and the precision of the effect estimates, especially for $p f m d r 1$ haplotypes. Another limitation is that not all predictors of recrudescence were measured, such as pharmacokinetics, host genetics, immunity, and nutritional status.

Molecular markers of drug-resistant malaria, such as pfmdr1, are promising tools for the surveillance of drug resistance. $p f m d r 1$ copy number is not only strongly associated with recrudescence to artesunate-mefloquine but also with Coartem failure (artemether-lumefantrine). ${ }^{39}$ Surveillance for increased pfmdrl copy number could aid malaria control efforts by pinpointing areas where these drugs may be failing.

Received September 12, 2006. Accepted for publication January 1, 2007.

Acknowledgments: We thank the study subjects for their participation. We appreciate the assistance and support provided by Dr. Socheat Duong, Augustina Ika Susanti, Jesse Kwiek, William Miller, Anne Purfield, Paul Wilson, Nareth Kong, Wini Kania, and Dr. J. Kevin Baird. We thank MR4 for providing us with malaria DNA.

Financial support: This work was funded by the United States Navy Medical (NHRC/NMRC) ILIR Program.

Disclaimer: The views and opinions are those of the authors and do not purport to represent those of the US Navy or Department of Defense.

Authors' addresses: Alisa P. Alker, Naman K. Shah, and Steven R. Meshnick, Department of Epidemiology, UNC Chapel Hill School of Public Health, CB \# 7435, Chapel Hill, NC 27599, E-mails: alker@unc.edu,naman@email.unc.edu, and meshnick@email. unc.edu. Pharath Lim, Rithy Sem, and Frederic Ariey, Institut Pasteur du Cambodge, 5 Monivong Boulevard, BP 983, Phnom Penh, Cambodia, E-mails: pharath@pasteur-kh.org, srithy@pasteur-kh.org, and fariey@pasteur-kh.org. Poravuth Yi, National Center for Parasitology, Entomology and Malaria Control (CNM), 372 Boulevard Monivong/Rue 322, Phnom Penh, Cambodia, E-mail: yiporavuth@ yahoo.com. Denis Mey Bouth, World Health Organization, PO Box 1217, Phnom Penh, Cambodia, E-mail: denism@cam.wpro.who.int. 
Reiko Tsuyuoka, WHO Office in the Lao People's Democratic Republic, PO Box 343, Vientiane, Laos, E-mail: Tsuyuokar@lao. wpro.who.int. Jason D. Maguire, United States Naval Medical Research Unit No.2, U.S. Embassy, Jakarta, Indonesia, E-mail: JDMaguire@mar.med.navy.mil. Thierry Fandeur, Unité d'Immunologie Moléculaire des Parasites CNRS URA 2581, Institut Pasteur, 28 rue du Dr ROUX, 75724 Paris Cedex 15, France, E-mail: tfandeur@pasteur.fr. Chansuda Wongsrichanalai, National Institute of Public Health/Naval Medical Research Unit No. 2 Laboratory, Phnom Penh, Cambodia, E-mail: chansuda@namru2.med.navy.mil.

Reprint requests: Steven R. Meshnick, Department of Epidemiology, UNC Chapel Hill School of Public Health, CB \# 7435, Chapel Hill, NC 27599. E-mail: meshnick@email.unc.edu.

\section{REFERENCES}

1. Payne D, 1987. Spread of chloroquine resistance in Plasmodium falciparum. Parasitol Today 3: 241-246.

2. Fontanet AL, Johnson BD, Walker AM, Bergqvist Y, Hellgrn U, Rooney W, 1994. Falciparum malaria in eastern Thailand: a randomized trial of the efficacy of a single dose of mefloquine. Bull World Health Organ 72: 73-78.

3. Yeung S, Pongtavornpinyo W, Hastings IM, Mills AJ, White NJ, 2004. Antimalarial drug resistance, artemisinin-based combination therapy, and the contribution of modeling to elucidating policy choices. Am J Trop Med Hyg 71: 179-186.

4. World Health Organization, 2005. Susceptibility of Plasmodium falciparum to Antimalarial Drugs: Report on Global Monitoring: 1996-2004. Geneva: World Health Organization.

5. Vijaykadga S, Rojanawatsirivet C, Cholpol S, Phoungmanee D, Nakavej A, Wongsrichanalai C, 2006. In vivo sensitivity monitoring of mefloquine monotherapy and artesunate-mefloquine combinations for the treatment of uncomplicated falciparum malaria in Thailand in 2003. Trop Med Int Health 11: 211-219.

6. Mey Bouth D, Tsuyuoka R, Poravuth Y, Narann TS, Seila S, Lim C, Incardona S, Lim P, Sem R, Socheat D, Christophel E, Ringwald P, 2006. Surveillance of the efficacy of artesunate and mefloquine combination for the treatment of uncomplicated falciparum malaria in Cambodia. Trop Med Int Health 11: $1360-1366$.

7. Bloland PB, Ettling M, 1999. Making malaria-treatment policy in the face of drug resistance. Ann Trop Med Parasitol 93: 5-23.

8. Olliaro P, 2005. Drug resistance hampers our capacity to roll back malaria. Clin Infect Dis 41: S247-S257.

9. Khim N, Bouchier C, Ekala MT, Incardona S, Lim P, Legrand E, Jambou R, Doung S, Puijalon OM, Fandeur T, 2005. Countrywide survey shows very high prevalence of Plasmodium falciparum multilocus resistance genotypes in Cambodia. Antimicrob Agents Chemother 49: 3147-3152.

10. Duraisingh MT, Roper C, Walliker D, Warhurst DC, 2000. Increased sensitivity to the antimalarials mefloquine and artemisinin is conferred by mutations in the pfmdr1 gene of Plasmodium falciparum. Mol Microbiol 36: 955-961.

11. Pickard AL, Wongsrichanalai C, Purfield A, Kamwendo D, Emery K, Zalewski C, Kawamoto F, Miller RS, Meshnick SR, 2003. Resistance to antimalarials in Southeast Asia and genetic polymorphisms in pfmdr1. Antimicrob Agents Chemother 47: 2418-2423.

12. Price RN, Cassar C, Brockman A, Duraisingh M, van Vugt M, White NJ, Nosten F, Krishna S, 1999. The pfmdr1 gene is associated with a multidrug-resistant phenotype in Plasmodium falciparum from the western border of Thailand. Antimicrob Agents Chemother 43: 2943-2949.

13. Nelson AL, Purfield A, McDaniel P, Uthaimongkol N, Buathong N, Sriwichai S, Miller RS, Wongsrichanalai C, Meshnick SR, 2005. pfmdr1 genotyping and in vivo mefloquine resistance on the Thai-Myanmar border. Am J Trop Med Hyg 72: 586-592.

14. Reed MB, Saliba KJ, Caruana SR, Kirk K, Cowman AF, 2000. Pgh1 modulates sensitivity and resistance to multiple antimalarials in Plasmodium falciparum. Nature 403: 906-909.

15. Sidhu AB, Valderramos SG, Fidock DA, 2005. pfmdr1 mutations contribute to quinine resistance and enhance mefloquine and artemisinin sensitivity in Plasmodium falciparum. Mol Microbiol 57: 913-926.
16. Cowman AF, Galatis D, Thompson JK, 1994. Selection for mefloquine resistance in Plasmodium falciparum is linked to amplification of the $p f m d r 1$ gene and cross-resistance to halofantrine and quinine. Proc Natl Acad Sci USA 91: 1143-1147.

17. World Health Organization, 2003. Assessment and Monitoring of Antimalarial Drug Efficacy for the Treatment of Uncomplicated Falciparum Malaria. Geneva: World Health Organization.

18. Farnert A, Arez AP, Babiker HA, Beck HP, Benito A, Bjorkman A, Bruce MC, Conway DJ, Day KP, Henning L, MercereauPuijalon O, Ranford-Cartwright LC, Rubio JM, Snounou G, Walliker D, Zwetyenga J, do Rosario VE, 2001. Genotyping of Plasmodium falciparum infections by PCR: A comparative multicentre study. Trans $R$ Soc Trop Med Hyg 95: 225-232.

19. Durrand V, Berry A, Sem R, Glaziou P, Beaudou J, Fandeur T, 2004. Variations in the sequence and expression of the Plasmodium falciparum chloroquine resistance transporter (Pfcrt) and their relationship to chloroquine resistance in vitro. $\mathrm{Mol}$ Biochem Parasitol 136: 273-285.

20. Purfield A, Nelson A, Laoboonchai A, Congpuong K, McDaniel P, Miller RS, Welch K, Wongsrichanalai C, Meshnick SR, 2004. A new method for detection of pfmdr1 mutations in Plasmodium falciparum DNA using real-time PCR. Malaria J 3: 9.

21. Price RN, Uhlemann AC, Brockman A, McGready R, Ashley E, Phaipun L, Patel R, Laing K, Looareesuwan S, White NJ, Nosten F, Krishna S, 2004. Mefloquine resistance in Plasmodium falciparum and increased pfmdr1 gene copy number. Lancet 364: 438-447.

22. Ferreira ID, do Rosario VE, Cravo PV, 2006. Real-time quantitative PCR with SYBR Green I detection for estimating copy numbers of nine drug resistance candidate genes in Plasmodium falciparum. Malaria J 5: 1.

23. Wellems TE, Panton LJ, Gluzman IY, do Rosario VE, Gwadz RW, Walker-Jonah A, Krogstad DJ, 1990. Chloroquine resistance not linked to mdr-like genes in a Plasmodium falciparum cross. Nature 345: 253-255.

24. Collett D, 2003. Modelling Survival Data in Medical Research. Boca Raton, FL: CRC Press.

25. Poole C, 2001. Low p-values or narrow confidence intervals: Which are more durable? Epidemiology 12: 291-294.

26. Greenland S, Pearl J, Robins JM, 1999. Causal diagrams for epidemiologic research. Epidemiology 10: 37-48.

27. Greenland S, 1999. Re: Confidence limits made easy: interval estimation using a substitution method. Am J Epidemiol 149: 884.

28. Price RN, Nosten F, Luxemburger C, van Vugt M, Phaipun L, Chongsuphajaisiddhi T, White NJ, 1998. Artesunate/ mefloquine treatment of multi-drug resistant falciparum malaria. Trans $R$ Soc Trop Med Hyg 91: 574-577.

29. Mawili-Mboumba DP, Kun JF, Lell B, Kremsner PG, Ntoumi F, 2002. Pfmdr1 alleles and response to ultralow-dose mefloquine treatment in Gabonese patients. Antimicrob Agents Chemother 46: 166-170.

30. Pillai DR, Hijar G, Montoya Y, Marouino W, Ruebush TK 2nd, Wongsrichanalai C, Kain KC, 2003. Lack of prediction of mefloquine and mefloquine-artesunate treatment outcome by mutations in the Plasmodium falciparum multidrug resistance 1 (pfmdr1) gene for P. falciparum malaria in Peru. Am J Trop Med Hyg 68: 107-110.

31. Ittarat $\mathrm{W}$, Pickard $\mathrm{AL}$, Rattanasinganchan $\mathrm{P}$, Wilairatana $\mathrm{P}$, Looareesuwan S, Emery K, Low J, Udomsangpetch R, Meshnick SR, 2003. Recrudescence in artesunate-treated patients with falciparum malaria is dependent on parasite burden not on parasite factors. Am J Trop Med Hyg 68: 147-152.

32. Lim P, Chim P, Sem R, Nemh S, Poravuth Y, Lim C, Seila S, Tsuyuoka R, Denis MB, Socheat D, Fandeur T, 2005. In vitro monitoring of Plasmodium falciparum susceptibility to artesunate, mefloquine, quinine and chloroquine in Cambodia: 20012002. Acta Trop 93: 31-40.

33. Karbwang J, White NJ, 1990. Clinical pharmacokinetics of mefloquine. Clin Pharmacokinet 19: 264-279.

34. Teja-Isavadharm P, Watt G, Eamsila C, Jongsakul K, Li Q, Keeratithakul G, Sirisopana N, Luesutthiviboon L, Brewer TG, Kyle DE, 2001. Comparative pharmacokinetics and effect ki- 
netics of orally administered artesunate in healthy volunteers and patients with uncomplicated falciparum malaria. Am $J$ Trop Med Hyg 65: 717-721.

35. Hastings IM, Ward SA, 2005. Coartem (artemether-lumefantrine) in Africa: The beginning of the end? J Infect Dis 192: 1303-1304; author reply 1304-1305.

36. Denis MB, Kouznetsov RL, Giboda M, 1991. In vivo response of multi-resistant Plasmodium falciparum infections to mefloquine and its combination with sulfadoxine/pyrimethamine in Cambodia. Folia Parasitol (Praha) 38: 187-188.

37. Thimasarn K, Sirichaisinthop J, Vijaykadga S, Tansophalaks S, Yamokgul P, Laomiphol A, Palananth C, Thamewat U, Thaithong S, Rooney W, 1995. In vivo study of the response of Plasmodium falciparum to standard mefloquine/sulfadoxine/ pyrimethamine (MSP) treatment among gem miners returning from Cambodia. Southeast Asian J Trop Med Public Health 26: 204-212.

38. Stepniewska K, Taylor WR, Mayxay M, Price R, Smithuis F, Guthmann JP, Barnes K, Myint HY, Adjuik M, Olliaro P, Pukrittayakamee S, Looareesuwan S, Hien TT, Farrar J, Nosten F, Day NP, White NJ, 2004. In vivo assessment of drug efficacy against Plasmodium falciparum malaria: Duration of follow-up. Antimicrob Agents Chemother 48: 4271-4280.

39. Price RN, Uhlemann AC, van Vugt M, Brockman A, Hutagalung R, Nair S, Nash D, Singhasivanon P, Anderson T, Krishna S, White NJ, 2006. Molecular and pharmacological determinants of therapeutic response to artemether-lumefantrine in multidrug-resistant Plasmodium falciparum malaria. Clin Infect Dis 42: 1570-1577. 